\title{
Estimating psychometric functions in forced-choice situations: Significant biases found in threshold and slope estimations when small samples are used
}

\author{
J. K. O'REGAN and R. HUMBERT \\ Laboratoire de Psychologie Expérimentale, CNRS, Paris, France
}

\begin{abstract}
When a theoretical psychometric function is fitted to experimental data (as in the obtaining of a psychophysical threshold), maximum-likelihood or probit methods are generally used. In the present paper, the behavior of these curve-fitting methods is studied for the special case of forcedchoice experiments, in which the probability of a subject's making a correct response by chance is not zero. A mathematical investigation of the variance of the threshold and slope estimators shows that, in this case, the accuracy of the methods is much worse, and their sensitivity to the way data are sampled is greater, than in the case in which chance level is zero. Further, Monte Carlo simulations show that, in practical situations in which only a finite number of observations are made, the mean threshold and slope estimates are significantly biased. The amount of bias depends on the curve-fitting method and on the range of intensity values, but it is always greater in forced-choice situations than when chance level is zero.
\end{abstract}

In many applications, it is necessary to determine the relation between the frequency of an event and the intensity of an independent variable. The psychometric function describing such a relation can be obtained with empirical methods such as the method of constant stimuli, staircase methods, the method of limits, and adaptive methods. If more than simply the threshold value of the psychometric function is required-as, for example, when the slope of the function is of interest-it is necessary to fit a theoretical curve (often a cumulative normal or logistic function) to the data points. The choice of a particular function will depend on the experimenter's hypotheses, but the techniques used to fit the curve remain the same irrespective of the function chosen. The most general curve-fitting method is the method of maximum likelihood, in which the parameters of the psychometric function chosen are such that it is the one most likely to have generated the observed data.

Theoretical work on fitting psychometric functions (Berkson, 1955; Wetherill, 1963) has shown that the maximum-likelihood method is reliable and gives unbiased estimates of the psychometric function's parameters. But the work has been limited to situations in which chance level is zero-that is, in which the observable proportions of correct responses vary from 0 to 1 .

The first part of the present paper recalls these classic conclusions and extends them to the case in which chance level is not zero. This is the case for two-alternative (or

Correspondence may be addressed to J. K. O'Regan, Groupe Regard, Laboratoire de Psychologie Expérimentale, 28 rue Serpente, 75006 Paris, France. $n$-alternative) forced-choice procedures, for which chance level is $1 / 2$ (or $1 / n$ ). We also present theoretical estimations of the effects of sampling data in nonoptimal positions and over intensity ranges of different size.

In the second part of the paper, a computer simulation evaluates the applicability of the theoretical conclusions to realistic conditions, in particular to the case in which a small number of samples are taken. Both maximumlikelihood and probit methods, which are computationally less expensive, are investigated. Unfortunately, the surprising fact emerges that when chance level is nonzero, not only are variances larger, but significant biases are found in the estimators of both the slope and, more importantly, the threshold.

\section{ASYMPTOTIC VARIANCES}

The experimenter must decide what theoretical psychometric function to fit to a set of data. Signal detection theory assumes a cumulative Gaussian function (Green \& Swets, 1966); but the logistic function is similar, and, being easier to calculate, is often preferred (Cox, 1972). If $P[y(x)=1]$ is the probability of a correct response at the intensity $x$, the logistic function is

$$
P[y(x)=1]=1 /\{1+\exp [-b(x-a)]\} .
$$

This function varies from 0 to 1 and is completely determined by the two parameters $a$ and $b: a$ is the threshold value of $x$, where the probability of correct response is .5 (50\% threshold); $b$ characterizes the slope of the function at the threshold point (the slope is $b / 4$ ).

In the case in which chance level is not zero, the logistic function is modified to take the form 


$$
P[y(x)=1]=c+(1-c) /\{1+\exp [-b(x-a)]\} .
$$

Here, the function takes values between $c$ and 1 instead of between 0 and $1 . a$ is the threshold value of $x$, where the probability of correct response is $(1+c) / 2$-that is, halfway between chance level and $1 . b$ characterizes the slope of the function at the threshold point [the slope is $(1-c) b / 4]$.

Finding the logistic function that adequately describes an observed set of data reduces to finding estimators $\hat{a}$ and $\hat{b}$ of $a$ and $b$. This can be done with the method of maximum likelihood. In what follows, we will, without loss of generality, take the "true" values to be $a=0$ and $b=1$ [i.e., threshold at the intensity level $x=0$, and slope $(1-c) / 4$, when $x=0]$, and study the theoretical "asymptotic" variance of the estimators $\hat{a}$ and $b$-that is, the behavior of the quantities $n b^{2} V(\hat{a})$ and $n V(\hat{b})$ in the limit when the number $n$ of measurements taken at each intensity value is large. These quantities are normalized forms of the variances $V(\hat{a})$ and $V(\hat{b})$.

Classic research (Wetherill, 1963) has involved the study of the asymptotic variance of the maximumlikelihood estimators $\hat{a}$ and $\hat{b}$ for the case $c=0$. In these studies, $n$ measurements are assumed to be taken at each of two values of the intensity scale, $x_{1}$ and $x_{2}$. The results of the calculations (see Table 1, case $c=0$ ) show that the estimator $\hat{a}$ of the threshold has minimum normalized variance when both $x_{1}$ and $x_{2}$ are placed exactly at the threshold value. In a real experiment, in which the threshold is not known in advance, it is of course not possible to place measurements there. Nevertheless, one can try to place each new measurement near the threshold position by estimating that position from the preceding data. This is the principle of staircase and adaptive methods. Note, however, that, as shown in Table 1 , if all $2 n$ measurements were placed exactly at the threshold, the theoretical variance of the estimate of $b$ would be infinite. The table shows that to obtain the smallest normalized variance of $\hat{b}, n$ measurements should be obtained at each of the two $x$-positions corresponding to .08 and .92 correct response probabilities. Finally, to obtain the smallest variance for both $\hat{a}$ and $\hat{b}$, measurements must be placed at positions where response probabilities are .18 and .82 .

We have extended Wetherill's calculations to the case $c>0$. We calculated the functions giving the asymptotic variances for $\hat{a}$ and $b$ (see Appendix A). Then, by numeric methods, we determined the pair of sampling points that minimized these functions (see Table 1 , cases $c=1 / 4$, $1 / 3$, and $1 / 2$ ).

In the special case when $c=0$, we find the same result as did Wetherill. But as the chance level increases from zero, the theoretical variances of $\hat{a}$ and $\hat{b}$ increase. To minimize the variance of $\hat{a}$, data should still be sampled at the threshold position, $x=0$. However, to obtain the minimum variance for $\hat{b}$, the two positions where data should be sampled are no longer symmetrical about the threshold position, but are shifted to the regions of higher $x$ values. In addition, the points are closer together than they were in the case $c=0$.

\section{Inaccurate Positioning of Samples}

In experiments, since the threshold position and slope are not known in advance, data cannot be sampled exactly at the theoretically optimum positions. What error will be incurred by sampling in slightly nonoptimum positions? The upper panel of Figure 1 shows the theoretical variance of $\hat{a}$ obtained for different chance levels when $n$ measurements are taken at each of two points symmetrically placed at $\pm(x-a)$ on either side of the true threshold $a$. The lower panel shows the variances of $\hat{b}$ when the pair of points sampled is shifted laterally from their optimum positions (but with optimum spacing maintained); the abscissa indicates the midpoint between the two positions sampled. Variances are expressed in their normalized forms, $n b^{2} V(a)$ and $n V(b)$.

Figure 1 shows, as does Table 1 , that the variance is larger when $c>0$ than when $c=0$. Figure 1 also shows that the effect of inaccurately positioning samples is more

Table 1

Optimum Sampling Positions to Determine $a, b$, or Both

\begin{tabular}{cllllllllc}
\hline & $x_{1}$ & $x_{2}$ & $P x_{1}$ & $P x_{2}$ & Center & $x_{2}-x_{1}$ & $n b^{2} V(a)$ & $n V(b)$ & $n^{2} b^{2} V(a) V(b)$ \\
\hline$c=0$ & & & & & & & & & \\
$a$ & 0 & 0 & .5 & .5 & 0 & 0 & 4 & $\infty$ & $\infty$ \\
$b$ & -2.4 & 2.4 & .083 & .916 & 0 & 4.8 & 13.11 & 2.28 & 29.85 \\
$a, b$ & -1.54 & 1.54 & .176 & .823 & 0 & 3.08 & 6.88 & 2.90 & 19.95 \\
$c=1 / 4$ & & & & & & & & & \\
$a$ & 0 & 0 & .625 & .625 & 0 & 0 & 6.60 & $\infty$ & $\infty$ \\
$b$ & -1.43 & 2.69 & .394 & .952 & 0.63 & 4.12 & 20.41 & 4.74 & 96.84 \\
$a, b$ & -0.82 & 2.12 & .479 & .919 & 0.65 & 2.94 & 12.47 & 5.59 & 69.84 \\
$c=1 / 3$ & & & & & & & & & \\
$a$ & 0 & 0 & .666 & .666 & 0 & 0 & 8 & $\infty$ & $\infty$ \\
$b$ & -1.33 & 2.75 & .472 & .959 & 0.71 & 4.08 & 24.39 & 5.72 & 139.55 \\
$a, b$ & -0.74 & 2.24 & .548 & .936 & 0.75 & 2.98 & 15.37 & 6.75 & 102.16 \\
$c=1 / 2$ & & & & & & & & & \\
$a$ & 0 & 0 & .75 & .75 & 0 & 0 & 12 & $\infty$ & $\infty$ \\
$b$ & -1.19 & 2.85 & .616 & .973 & 0.83 & 4.04 & 36.33 & 8.50 & 308.84 \\
$a, b$ & -0.62 & 2.42 & .675 & .959 & 0.9 & 3.04 & 23.82 & 9.70 & 231.07 \\
\hline
\end{tabular}



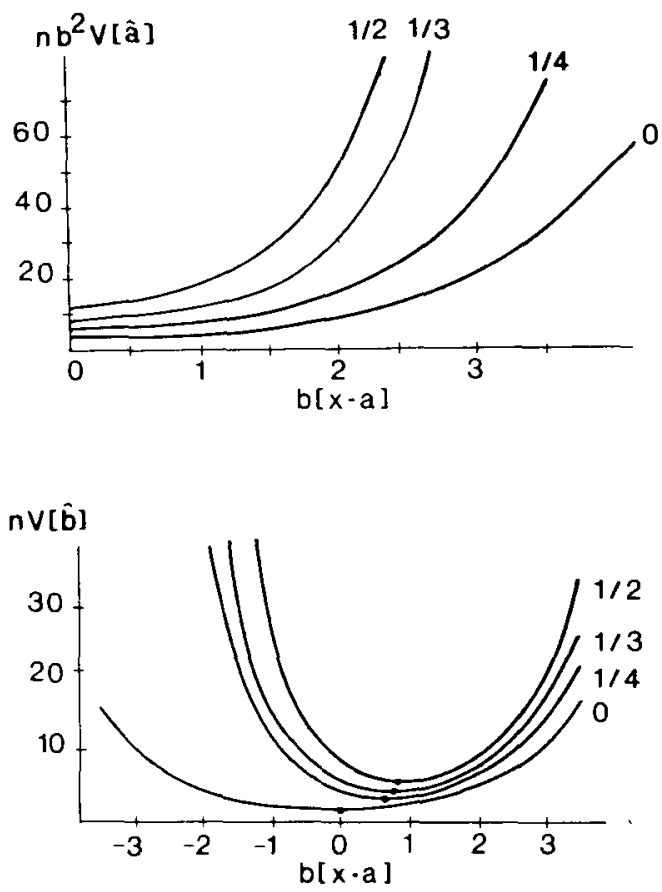

Figure 1. Upper graph: The effect of changing the size of the range of intensity values sampled on the asymptotic variance of the threshold estimate $\hat{a}$. The asymptotic variance, expressed in normalized form $n b^{2} V(\hat{a})$, is given as a function of the positions of points sampled on the psychometric function: $n$ values are assumed to have been sampled at each of two points placed symmetrically at a distance $\pm(x-a)$ on each side of the threshold intensity $a$. The abscissa gives the distance $(x-a)$ used. Because the curves must be symmetric, only the values for $(x-a)>0$ are shown. The different curves are for different chance levels. Lower graph: The effect of shifting the sampling range on asymptotic variance of the slope estimator b. Asymptotic variance, expressed in normalized form $n V(b)$, is given as a function of the midpoint of the range of intensity values sampled: $\boldsymbol{n}$ values are assumed to have been sampled at each of two positions situated at the two extremes of a range of optimum size (see Table 1), but centered at an intensity value at a distance $(x-a)$ from the threshold position. The different curves correspond to different chance levels. The dot on each curve shows the optimum sampling position for each chance level.

critical when $c>0$ : Whereas, when $c=0$, there is a plateau of approximately constant variance around the optimum position, the plateau is less marked for $c>0$, especially for $b$. Thus, when $c$ is nonzero, estimates of $b$ will be less stable than estimates of $a$. Finally, it is apparent that for $\hat{b}$, variances increase faster when the sampling positions are shifted left with respect to the optimum position.

\section{Sampling over a Range of Intensities}

In experiments, data will usually be sampled at more than one or two positions on the intensity scale over a range of positions that are thought a priori to include the threshold intensity. It would be interesting to know whether a large or a small range is preferable, and whether it matters if the range is not symmetrically placed with respect to the true threshold value. For this reason, asymptotic variances were calculated for a number of such cases.

We chose three ranges that were symmetrical about the true threshold $x=a$, and two asymmetrical ranges. The symmetrical ranges were: large (from $x-a=-5$ to +5 ), central (from $x-a=-1$ to +1 ), and external (this range included both the interval from $x-a=-2$ to -1 and the interval from $x-a=+1$ to +2 , but not the central region -1 to +1 ; it was included to see if it would give better combined accuracy of threshold and slope). The ranges that were asymmetrical about the true threshold were: shifted to the right (from $x-a=0$ to +2 ), and shifted to the left (from $x-a=-2$ to 0 ). For each range, measurements were taken at 10 equally spaced intensity levels, including the limits. The logistic function in Equation 2 was again assumed to generate the data, and $a=0$ and $b=1$ were taken, without loss of generality. Asymptotic variances of the estimates of $a$ and $b$ were obtained in the normalized forms $n b^{2} V(\hat{a})$ and $n V(\hat{b})$, using Formulas A1 and $A 2$ of Appendix $A$, where $n$ is the number of measurements taken at each of the intensity levels. Chance levels $0,1 / 3$, and $1 / 2$ were considered.

Figure 2 shows the results of the calculations. As before, variance increases with chance level (the variances for $c=1 / 2$ are four to five times greater than those for $c=0$ ). As expected from the data in Table 1 , the curves also show that, among the sampling regions considered, the best region for accurate estimates of $a$ is the central $[-1,1]$ range, because sampling is closest to the optimum position near threshold. In the case of $b$, the best ranges among those considered here were the large $[-5,5]$ and the external $[-2,1][1,2]$ range.

\section{MONTE CARLO SIMULATION FOR SMALL SAMPLES}

The asymptotic variances calculated above are theoretical lower limits for the variance expected when the number of measurements $n$ made at each intensity value is large. But in practical situations, only $n=10$ or $n=20$ measurements may be made at each intensity level. How do the estimators $\hat{a}$ and $\hat{b}$ behave for such small samples?

In the following Monte Carlo simulations, the same chance levels, ranges of intensity, and number of intensity values were used as in calculating asymptotic variances. At each of the $N=10$ intensity values, the "true" value of the success probability $p$ expected at that intensity was determined from the logistic function in Equation 2 with $a=0$ and $b=1$. Then, $n=10$ simulated Bernoulli measurements were taken at that intensity value, using the probability $p$ of success. This was done by drawing a pseudorandom number in the range $[0,1)$ and recording either a "success" if the number was less than $p$, or a "failure" otherwise. A logistic curve was then fitted through all the simulated data points ( $n=10$ measurements at $N=10$ intensity values), providing one pair of estimates $\hat{a}$ and $\hat{b}$ of the "true" values $a=0$ and $b=1$. The whole curve-fitting process was repeated 500 times, and the 

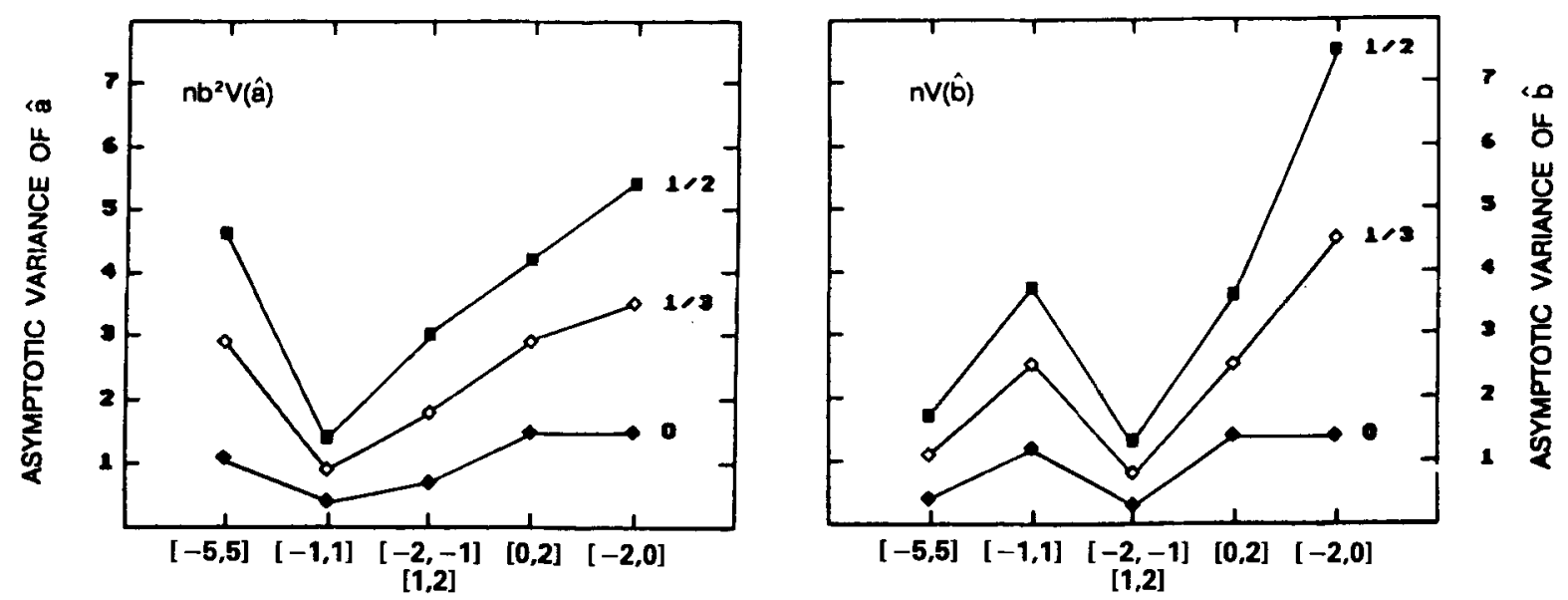

\section{RANGE OF INTENSITIES SAMPLED}

Figure 2. Asymptotic variances of estimators $\hat{a}$ and $\hat{b}$ for different chance levels, as a function of the range of intensity values sampled. $n=10$ data samples were taken at $N=10$ equally spaced positions within each range indicated. The variances are expressed in their normalized forms, $n b^{2} V(a)$ for $a$ and $n V(b)$ for $b$. Note that, for clarity, the points for a given chance level have been connected even though there is no logical relation between the different intensity ranges indicated on the abscissa.

mean and variance of the 500 observed values of $\hat{a}$ and $\hat{b}$ were calculated.

Both maximum-likelihood and probit methods were used to fit the curves. The probit method is an often-used alternative for the maximum-likelihood method (which requires time-consuming calculations): The data are first linearized with an inverse cumulative normal distribution, and then a straight line is fitted to them using the method of least squares (Finney, 1947). Berkson (1955) shows that this method is generally equivalent to the method of maximum likelihood, even if only few measurements are made at each intensity value. The probit method used in the present study was an adaptation of the algorithm described by Lieberman (1983). Instead of an inverse cumulative normal distribution, an inverse logistic function was used, since the underlying data was generated by a logistic function. It would have been more reasonable to call the method the "logit" method rather than the "probit" method for this reason, but we will retain the more common term "probit."

The maximum-likelihood method used in the present simulation was very similar to the algorithm used by Watson and Pelli (1983). The search for the maximum of the likelihood function was done exhaustively within the intensity range -5 to +5 , in steps of 0.5 . This means that the maximum and minimum values of the estimated threshold could never be outside this range. Similarly, the estimated values of $b$ were limited to the range 0.2 to 2 in steps of 0.2. In the case in which the same maximum value was attained by a number of values of $a$ and $b$, the first pair found was used.

The variances of the observed estimates of $a$ and $b$ are plotted in Figure 3. They give an evaluation of the variability of the fits provided by the probit and maximum- likelihood methods. The appropriate asymptotic variances to which these data should be compared are given in Figure 2, except that because $n=10$ data points were used at each intensity level in the Monte Carlo simulation, the asymptotic variances shown in Figure 2 should be divided by 10 . When this is done, we see that as expected, the small sample variances are always larger than the asymptotic ones. One difference between the predicted and observed variances is the fact that the minimum variance for $\hat{a}$ was expected for the central range $[-1,1]$. However, in the simulation, the minimum was obtained for the large $[-5,5]$ and external $[-2,-1][1,2]$ ranges.

In the case of the probit method, the organization of the curves does not correspond exactly to the pattern predicted from the asymptotic variances, since for both $\hat{a}$ and $\hat{b}$ the curves for the different chance levels cross each other, whereas they did not in Figure 2.

We have until now looked only at the variances of $\hat{a}$ and $\hat{b}$, but in the small-sample case, we also asked how accurately the means of $\hat{a}$ and $\hat{b}$ over 500 simulations estimated the true values $a=0$ and $b=1$. These means are plotted in Figure 4. Surprisingly, they often deviated strongly from the true values. The statistical significance of these deviations can be evaluated through calculating their standard error over the 500 simulations that were performed. In many cases, the deviations are statistically significant.

The left-hand panels of Figure 4 show estimations of $a$ : For symmetrical ranges, maximum likelihood correctly estimates threshold (except for chance level 1/3, range $[-5,5]$, which is significantly lower than 0 ), whereas the probit method does so only for chance level 0 , with increasing underestimation as chance level increases. For asymmetric ranges, both methods give threshold estimates that 

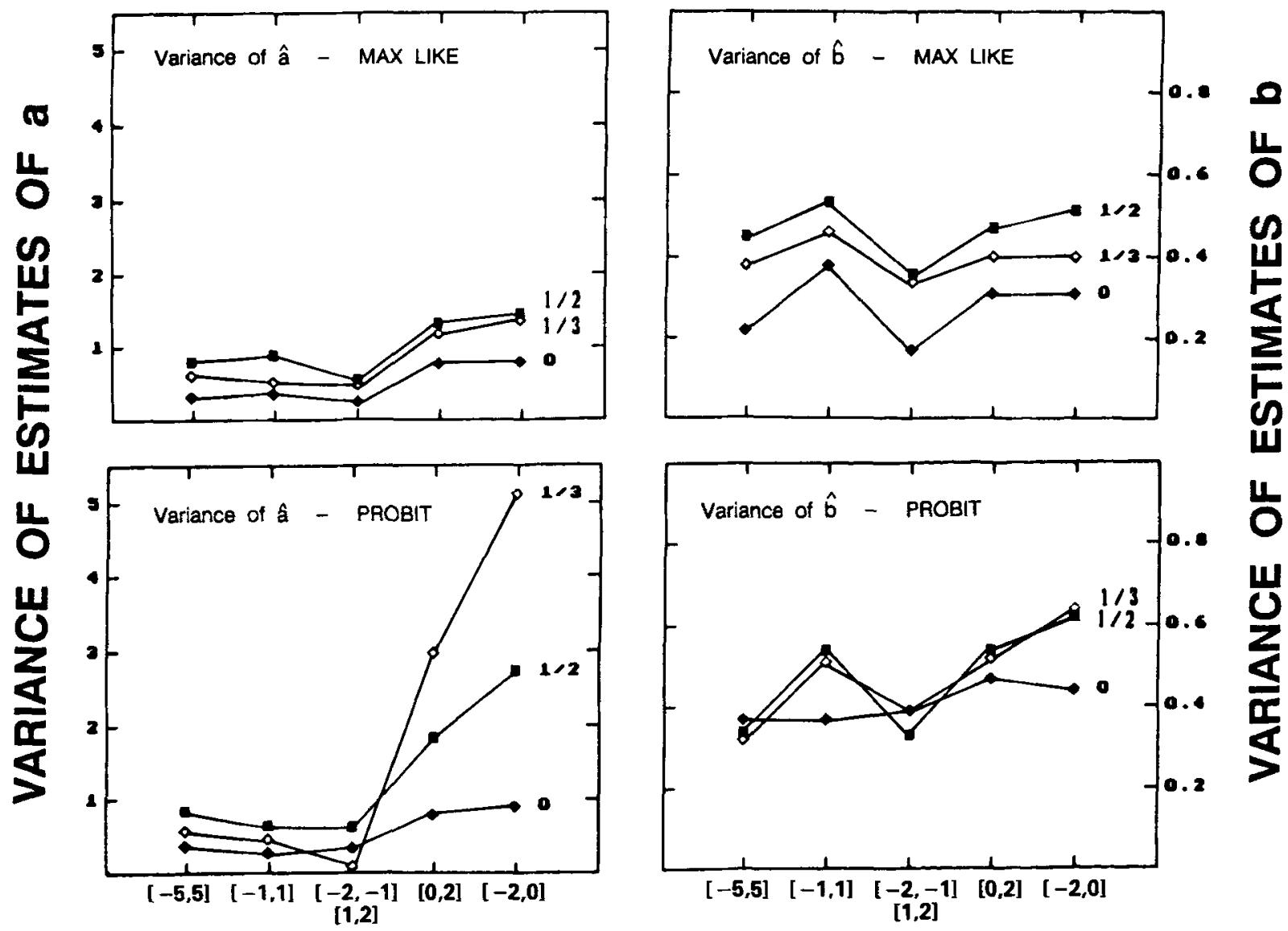

RANGE OF INTENSITIES SAMPLED

Figure 3. Variance of the extimators a and $\mathbf{6}$ obtained over $\mathbf{5 0 0}$ Monte Carlo simulations. For each simulation, a psychometric function was adjusted using the maximum-likelihood and the probit methods. The results are presented for the different chance levels as a function of the different test yanges used. $n=10$ simulated data values were taken from an assumed logistic function with $a=0$ and $b=1$ at $N=10$ equally spaced positions within each range indicated. Variance is now not in normalized form, but simply $V(a)$ and $V(b)$.

are strongly biased in a direction opposite to that of the range shift, with chance level playing no consistent role.

The right-hand panels of Figure 4 show estimations of $b$ : maximum likelihood produced values significantly different from $b=1$ excepting two cases ( $c=0$, central $[-1,1]$ and external $[-2,1][1,2]$ ranges), but the differences are not systematically related either to chance level or range. The probit method produces significantly different values only when $c=1 / 2$, systematically underestimating $b$.

\section{POSSIBLE CAUSE OF THE BIAS}

It is not completely surprising to find that in the smallsample case, the estimates of the slope of the psychometric function are biased-it seems to be known within psychophysics that stable slope measurements are difficult to obtain. However, the finding that even the estimate of threshold can be significantly biased is very troubling, since threshold measurements are ubiquitous in psychophysics. As a first step to understanding the source of both slope and threshold biases, we asked whether they arise from a uniform tendency toward a bias, or whether the estimation methods are generally accurate, but occasionally break down and give extreme values that cause the bias (as suggested by Watt \& Andrews, 1981). The latter situation would be less troublesome, since the occasional extreme values would be easily recogized and discounted.

However, consideration of the distributions of $\hat{a}$ and $\hat{b}$ showed that biases could not be attributed to extreme values. For $\hat{a}$, the modes of the distributions are always correct at $a=0$, and bias seems to arise because the distributions of the estimates of $\hat{a}$ are asymmetrical, with a longer tail on one side. This was confirmed with calculation of their third-order moments, which increased when bias increased.

The modes of the distributions of $b$ estimates are not at the correct value of $b=1$, and bias stems from the distributions' being laterally shifted. This is clear in the probit case. In the maximum-likelihood case, small piles of observations sometimes appear at one or the other end of the histograms, no doubt because only a limited range of $b$-values was considered in searching for the maximum of the maximum-likelihood function. The piles do not ap- 

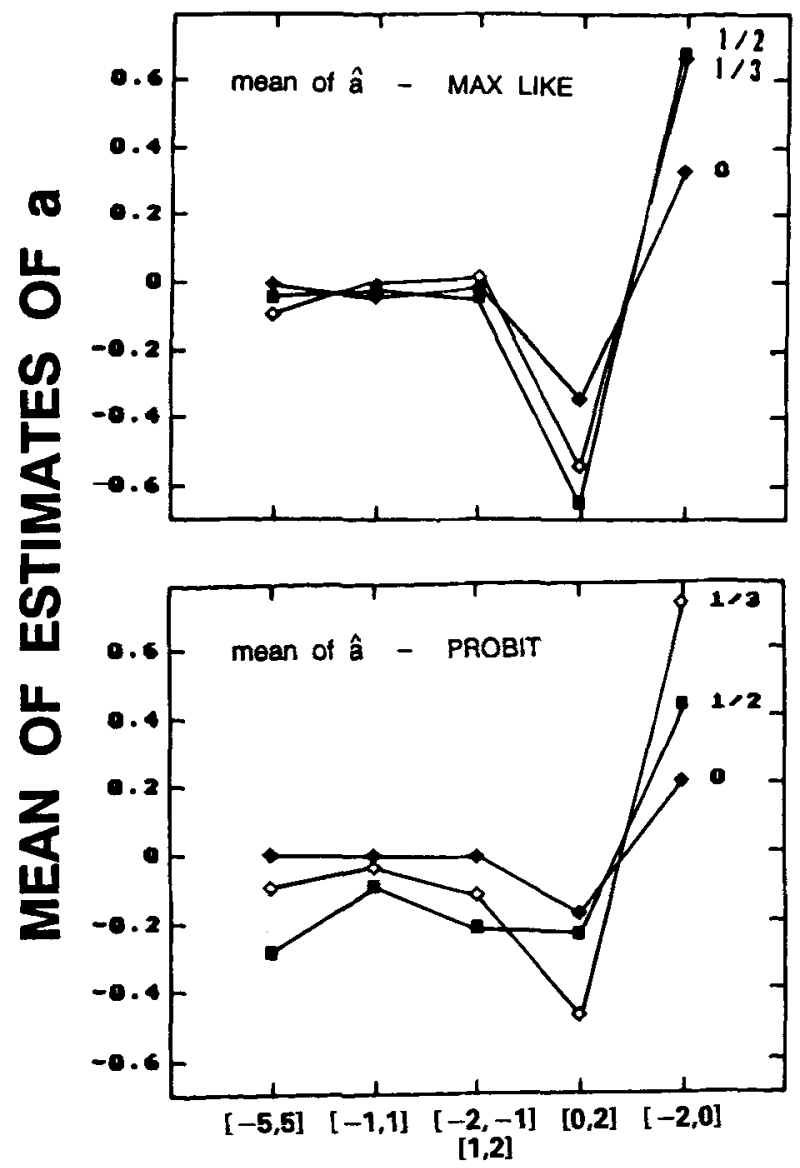

RANGE OF INTENSITIES SAMPLED

Figure 4. Means of the estimators $\hat{d}$ and $\hat{b}$ in the Monte Carlo simulations. Circled points deviate significantly (two-tailed $t$ test, $p<.001)$ from the true values $a=0$ and $b=1$.

pear to result from occasional extreme values, since they appear to be a natural continuation of the observed distributions into the large $b$ values. When the piles are ignored, the distributions are still shifted relative to the true value of $b=1$. (However, the variances were rendered more similar to the values expected from the asymptotic calculations shown in Figure 2-that is, the curves no longer crossed each other.)

Since the biases are not caused by occasional extreme values, perhaps they are generated by the approximations inherent in the implementation of the algorithms. Different problems arose in the probit and the maximumlikelihood cases.

In the probit case, curve fitting was carried out by first calculating total percent correct over the $n=10$ measurements at each intensity value, and then transforming the resulting scores by means of an inverse logistic function. In the case in which the 10 measurements were all correct or all false, the inverse transform would give an intensity value of plus or minus infinity. For the purposes of the subsequent least squares curve fitting, these values had either to be replaced by some finite values, say 5 and

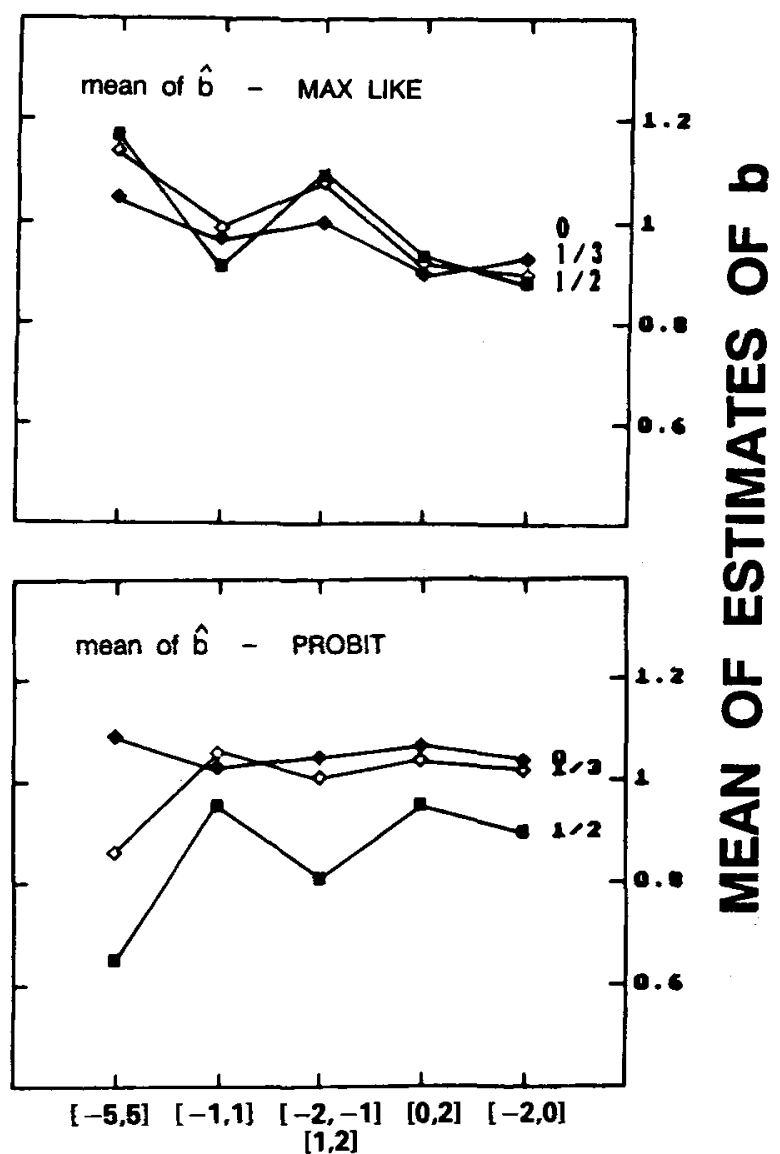

-5 , or to be ignored. Because the first possibility seemed at first sight to be more arbitrary, it was decided to adopt the solution of ignoring cases in which the inverse logistic transform became infinite. It is conceivable that the biases observed in the probit analysis were related to these problems. However, we also did simulations in which infinite values were replaced by the values -3 and +3 , or -5 and +5 , or -10 and +10 . The biases in the obtained threshold estimates were modified by these choices, but they remained statistically significant, suggesting that the source of the bias did not lie here. Nevertheless, in the discussion below of the effect of sample size on the behavior of the probit method, we shall see that the biases are related in another way to the problem of the infinite values.

In the case of the maximum-likelihood method, the maximum of the likelihood function was searched for by sampling the function on a fairly coarse basis: values of $a$ were sampled in steps of 0.5 between -5 and +5 , values of $b$ in steps of 0.2 between 0.2 and 2.0 . In the case in which the function had a plateau, the smallest $a$ and $b$ values were taken. It is conceivable that the biases in 
threshold and slope estimates were related to these problems. However, the following discussion will suggest that the main source of bias lies elsewhere.

Another possibility for explaining the biases in threshold and slope estimations might lie in the small-sample behavior of the maximum-likelihood and probit estimation methods. If this is so, biases should disappear when larger sample sizes are used. We repeated the simulations in both probit and maximum-likelihood methods with $n=100$ instead of $n=10$ samples per intensity value. The biases in the estimations of $a$ and $b$ for $c>0$ decreased, disappearing entirely for the symmetrical intensity ranges. When $n=1,000$ samples per intensity were used, the bias disappeared also in the shifted ranges. For example, using the maximum-likelihood method on the range $[0,2]$ with $c=1 / 2$ (one of the worst cases-see Figure 4), in 500 simulations, the "correct" threshold of $a=0$ was found in all 500 cases, and the mean of the estimates of $b$ was 1.001 , with an extremely small standard deviation (0.015).

How could bias arise out of the small-sample behavior? Appendix B and Appendix C are first steps toward explanations for the probit and maximum-likelihood methods, respectively. It appears that the problem arises in the probit case because the inverse logistic function is meaningless when trials at a given intensity value turn out to be all $1 \mathrm{~s}$ or all $0 \mathrm{~s}$. The probability that this occurs tends to zero when the number $n$ of samples taken at each intensity value is large, but it is nonnegligible when $n$ is small. Whichever way this problem is dealt with in a particular numerical implementation of the probit method, be it by replacing the problematic values by some arbitrary finite value, or by ignoring them, systematic biases will result. These biases will be strongest when chance level is nonzero, because more cases of all $1 \mathrm{~s}$ or all 0 s are likely to occur. The size of the bias will also be influenced by the region of intensity values within which measurements are taken, with bias getting stronger as we leave the region where the probability of success is near $1 / 2$.

In the case of the maximum-likelihood method, the argument in Appendix $\mathrm{C}$ suggests that the expected values of the estimators $\hat{a}$ and $\hat{b}$ may be undefined if only a small number of samples are taken at each intensity value. Again, the reason appears to be related to the occurrence of cases where all $1 \mathrm{~s}$ or all $0 \mathrm{~s}$ are observed at a given intensity value. These can be avoided if one makes many measurements per intensity value, keeps them to the region where the probability of success is near 0.5 , and uses chance level zero.

The above considerations show that to avoid biases, sampling must be as near as possible to the position at which $p=.5$, and symmetrically placed around this position. (Of course when $c=1 / 2$, this position is at $-\infty$, and biases become inevitable.) But a second point must be noted. Reducing bias is not the only condition necessary for reliable estimates, since we must also be sure that the variance of the estimates is reasonable. The data on asymptotic variance and the Monte Carlo simulations show that to minimize the variance, sampling must be done symmetrically about the threshold value. Unfortunately, only in the case $c=0$ does the threshold position also correspond with the place at which bias becomes negligible!

\section{CONCLUSION}

We have shown that when small samples are used (10 per intensity value), maximum-likelihood and probit methods are not satisfactory for estimating the threshold and slope of a psychometric function, particularly in the case in which chance level is nonzero (i.e., in $\boldsymbol{n}$-alternative forced-choice situations), since they can give systematic biases. To avoid bias in the threshold and slope estimates, sampling must be done close to the point at which $p=.5$, and a sufficient number of samples must be taken at each intensity value so that the probability of obtaining all successes or all failures is negligible. In the case of the $n$-alternative forced-choice procedure, this is harder to ensure than when chance level is zero, and in the case of the two-alternative forced choice, it is actually impossible, since $p=.5$ occurs at an intensity value of $-\infty$ !

These findings corroborate the results of McKee, Klein, and Teller (1985), who found that for small sample sizes, the probit technique gives systematic biases in the threshold estimation in two-alternative forced-choice situations.

An important consequence of our findings concerns the reliability of the now ubiquitous adaptive (or sequential) algorithms used for measuring psychophysical functions. At each step in such algorithms, either explicitly or implicitly, an estimation of the psychophysical function is often made, using maximum-likelihood or probit techniques. If this estimation is biased (which is likely, if the chance level is not zero), errors may build up progressively, and the final estimates obtained may be erroneous. Thus, Emerson (1986) did Monte Carlo simulations of sequential estimation procedures for a two-alternative forced-choice task (for which chance level is $1 / 2$ ). Each new measurement was placed at the threshold position estimated from the preceding measurements. When a maximum-likelihood method was used to estimate this position, strong biases were observed in the final estimated threshold. Similarly, in Hall's (1981) study, in which the chance level was $1 / 4$, sequentially estimated parameters were biased. Hall used a staircase method in which the placement of each new measurement did not depend on the previous estimation of the psychometric function. However, the final result of each simulation was obtained by fitting a psychometric curve to all points obtained during that simulation. On the other hand, as was expected from our results, adaptive estimates of psychophysical function parameters were unbiased in the case of Findlay's (1978) simulation, since he used a chance level of zero. The question of the bias expected from nonparametric staircase methods that do not either explicitly or implicitly make use of curve-fitting methods (e.g., Wetherill \& Levitt, 1965) remains open, but it is possible that these have no bias. Emerson (1986) suggested an alternative, 
Bayesian, method of estimating the psychophysical function, which worked better than the maximum-likelihood method when used in simulations of an adaptive sequential procedure. However, Emerson did not directly evaluate the small-sample curve-fitting accuracy of his method, nor did he attempt to use it to simultaneously estimate the values of both $a$ and $b$. It would be interesting to repeat the kind of calculations presented in the present article with Emerson's Bayesian method.

In any case, it seems clear that maximum-likelihood and probit estimation techniques should not be used in psychophysical procedures (adaptive or not), particularly when chance level is greater than zero, unless the number of samples per intensity value is considerably greater than 10 .

\section{REFERENCES}

Berkson, J. (1955). Maximum likelihood and minimum chi-square estimates of the logistic function. Joumal of the American Statistics Association, 50, 130-162.

Cox, D. R. (1972). Analyse des donnés binaires. Paris: Dunod.

EMERSON, P. L. (1986). Observations on maximum-likelihood and Bayesian methods of forced-choice sequential threshold estimation. Perception \& Psychophysics, 39, 151-153.

FindLAY, J. M. (1978). Estimates on probability functions, a more virulent PEST. Perception \& Psychophysics, 23, 181-185.

Finney, D. J. (1947). Probit analysis. Cambridge, England: Cambridge University Press.

GreEN, D. M., \&WETS, J. A. (1966). Signal detection theory and psychophysics. New York: Wiley.

HALL, J. L. (1981). Hybrid adaptive procedure for estimation of psychometric functions. Journal of the Acoustical Society of America, 69, 1763-1769.

Lieberman, H. R. (1983). Computation of psychophysical thresholds using the probit technique. Behavior Research Methods \& Instrumentation, 15, 446-448.

MCKeE, S. P., KLeIN, S. A., \& Teller, D. Y. (1985). Statistical properties of forced-choice psychometric functions: Implications of probit analysis. Perception \& Psychophysics, 37, 286-298.

Watson, A. B., \& Pellu, D. G. (1983). QUEST: A Bayesian adaptive psychometric method. Perception \& Psychophysics, 33, 113-120.

WATT, R. J., \& ANDREWS, D. P. (1981). A.P.E: Adaptive probit estimation of psychometric functions. Current Psychological Reviews, 1, 205-214.

WeTHERILL, G. B. (1963). Sequential estimation of quantal response curves. Journal of the Royal Statistics Society, B25, 1-48.

WETHERILL, G. B., \& LeVITT, H. (1965). Sequential estimation of points on a psychometric function. British Joumal of Mathematical \& Statistical Psychology, 18, 1-10.

\section{APPENDIX A \\ Maximum-Likelihood Technique}

\section{General Principle}

Assume experimental conditions in which the probability of a correct response at intensity level $x_{i}$ is given by the logistic function

$$
P\left(x_{i}\right)=c+\frac{1-c}{\left\{1+e^{-b\left(x_{i}-a\right)}\right\}},
$$

where $c$ is the chance level and $a$ and $b$ the parameters determining threshold and slope. The probability of an incorrect response is $1-P\left(x_{i}\right)$.
Assume in a given experiment that, at each intensity $x_{i}, n_{i}$ trials are made, and that they give $k_{i}$ correct responses and $n_{i}-k_{i}$ wrong responses. If $N$ different intensity levels are tested, we will obtain a sample of $N$ pairs $\left(x_{i}, k_{i}\right)$. At each intensity level, the distribution of correct responses is binomial, and, therefore, the probability of having obtained the observed sample is

$$
L=\prod_{i=1}^{N}\left[P\left(x_{i}\right)\right]^{k_{i}}\left[1-P\left(x_{i}\right)\right]^{n_{i}-k_{i}}
$$

or

$$
L^{\prime}=\log (\mathrm{L})=\sum_{i=1}^{N}\left\{k_{i} \log \left[P\left(x_{i}\right)\right]+\left(n_{i}-k_{i}\right) \log \left[1-P\left(x_{i}\right)\right]\right\} .
$$

$L^{\prime}$, the likelihood function, depends on the two parameters $a$ and $b$. The best estimators $\hat{a}$ and $\bar{b}$ of $a$ and $b$, given the observed set of responses, are those which maximize the likelihood function. They must therefore satisfy the equations

$$
\begin{aligned}
& \frac{\partial L^{\prime}}{\partial a}=0 \\
& \frac{\partial L^{\prime}}{\partial b}=0 .
\end{aligned}
$$

\section{Asymptotic Variances}

The calculation of asymptotic variances implies the calculation of the expectation of the second derivatives of the likelihood function:

$$
\begin{gathered}
E\left(\frac{\partial^{2} L^{\prime}}{\partial a^{2}}\right)=-(1-c) b^{2} \sum n_{i} \phi_{i} / h_{i} \\
E\left(\frac{\partial^{2} L^{\prime}}{\partial b^{2}}\right)=-(1-c) \sum n_{i}\left(x_{i}-a\right)^{2} \phi_{i} / h_{i} \\
E\left(\frac{\partial^{2} L^{\prime}}{\partial a \partial b}\right)=-(1-c) b \sum n_{i}\left(x_{i}-a\right) \phi_{i} / h_{i}
\end{gathered}
$$

where

$$
\phi_{i}=e^{-b\left(x_{i}-a\right)} /\left\{1+e^{-b\left(x_{i}-a\right)}\right\}^{2}
$$

and

$$
h_{i}=1+c \cdot e^{-b\left(x_{i}-a\right)} .
$$

Note that when $c=0$, the system becomes Wetherill's (1963) equations.

With these equations, we have for asymptotic variances:

$$
V(\hat{a})=-\Delta^{-1} E\left(\frac{\partial^{2} L^{\prime}}{\partial b^{2}}\right)
$$

and

$$
V(\hat{b})=-\Delta^{-1} E\left(\frac{\partial^{2} L^{\prime}}{\partial a^{2}}\right)
$$

where

$$
\Delta=E\left(\frac{\partial^{2} L^{\prime}}{\partial a^{2}}\right) E\left(\frac{\partial^{2} L^{\prime}}{\partial b^{2}}\right)-\left[E\left(\frac{\partial^{2} L^{\prime}}{\partial a \partial b}\right)\right]^{2}
$$

Following Wetherill (1963), we consider the situation in which observations are placed in two groups of equal size $n$, at inten- 
sities $x_{1}$ and $x_{2}$. We calculated by numeric methods the pair of values $\left(x_{1}, x_{2}\right)$ which minimized the variances $n b^{2} V(\hat{a}), n V(\hat{b})$, and $n^{2} b^{2} V(\hat{a}) V(\hat{b})$. The results are presented in Table 1 .

\section{APPENDIX B \\ Possible Cause of Bias in the Probit Method}

Assume that the "true" logistic function giving rise to the data is given by:

$$
p(x)=c+\frac{1-c}{1+e^{-B(x-A)}} .
$$

Suppose that $n$ samples are taken at intensity value $x$, and that $k$ come out as $1 \mathrm{~s}$, and $n-k$ as $0 \mathrm{~s}$. In the probit calculation, the proportion $k / n$ would be transformed by the inverse logistic transform to the value $\operatorname{logit}(k / n)$, where $\operatorname{logit}(p)$ is defined as

$$
\ln \left(\frac{p-c}{1-p}\right)
$$

and then used in a linear regression.

What is the expected value of $\operatorname{logit}(k / n)$, contributing to the regression calculation? The proportion $k / n$ will occur with probability

$$
[p(x)]^{k}[1-p(x)]^{n-k}\left(\begin{array}{c}
n \\
k
\end{array}\right) .
$$

The expected value of $\operatorname{logit}(k / n)$ is therefore:

$$
\mathrm{E}[\operatorname{logit}(k / n)]=\sum_{k=0}^{n}[p(\mathrm{k})]^{k}[1-p(k)]^{n-k}\left(\begin{array}{l}
n \\
k
\end{array}\right) \cdot \operatorname{logit}(k / n) .
$$

A problem arises here: The first few terms and the last term in this sum are infinite because the logit is undefined when $k \leq c n$ and $k=n$. The solution that can be adopted is to ignore these terms or replace them with some (arbitrary) large value. How will this affect the computation of the expected value? If $n$ is large, the binomial coefficient multiplying the inverse logistic function in the first and last terms will be small, and the error made may be minimal. But if $n$ is small, the coefficients will be appreciable, and a large error will be made. $\mathrm{Nu}$ merical calculations show that if the first and last terms are ignored, for the case $n=10$, the value of $E[\operatorname{logit}(k / n)]$ is approximately correct-that is, it is close to $\operatorname{logit}[\mathrm{E}(k / n)]=\operatorname{logit}[p(x)]$ $=x$-in the region near the threshold $x=0$ (where the probability of observing all $1 \mathrm{~s}$ or all $0 \mathrm{~s}$ is smallest). But calculations also show that $\mathrm{E}[\operatorname{logit}(k / n)]$ is progressively underestimated as $x$ increases above 0 , and overestimated as $x$ decreases below 0 . The rate at which the error increases as we leave the favorable region near threshold is much faster in the cases when $c$ is nonzero than in the case when $c$ is zero.

These considerations show that the probit method can only be accurate if a large number of samples per intensity value is taken, and if the samples are taken near threshold. These conditions ensure that the probability of obtaining all $1 \mathrm{~s}$ or all 0 s is not too large. The range of intensity values within which this will be possible is much smaller in the case when $c$ is nonzero than when $c$ is zero.

\section{APPENDIX C \\ Bias in the Maximum-Likelihood Method}

The following paragraphs contain a simplified problem, to show that in such a case bias can be expected. Suppose that the slope of the function is known a priori to be 1 , and only the threshold $a$ remains to be determined. Suppose $n$ samples are taken at a single intensity value $x$. Assume that the "true" underlying logistic function is

$$
p_{A}(x)=c+\frac{1-c}{1+e^{-B(x-A)}},
$$

so the "true" value of $a$ is $A$.

Assume that $k$ out of $n$ responses observed at $x$ gave response 1 , and that $n-k$ gave response 0 . The probability that this will have occurred if the underlying logistic function were

$$
p_{a}(x)=c+\frac{1-c}{1+e^{-B(x-a)}}
$$

would be given by the binomial probability

$$
\left[p_{a}(x)\right]^{k}\left[1-p_{a}(x)\right]^{n-k}\left(\begin{array}{c}
n \\
k
\end{array}\right)=L(a),
$$

which is the likelihood function, a function of $a$. We seek the value of $\hat{a}$ of $a$ that maximizes this function. From the theory of binomial terms (or by differentiation), it is clear that the function will be maximum when

$$
p_{a}(x)=\frac{k}{n}
$$

This is reasonable, because measurements are taken at a single intensity point, and so we expect the estimated logistic curve to pass through the value $p=k / n$ found at that point. From C3 we have

$$
\hat{a}=\left[x-\frac{\operatorname{logit}(k / n)}{B}\right] .
$$

Now what happens if the same experiment is repeated a number of times? Different values of $k$ will be found: $k_{1}, k_{2}, k_{3}, \ldots$, and from these, different estimates of $\hat{a}$ will be obtained by the method just outlined: $\hat{a}_{1}, \hat{a}_{2}, \hat{a}_{3}, \ldots$ Since $k$ is taken from a binomial distribution with success probability given by $\mathrm{C} 1$ above, we can calculate the expected value of $\hat{a}$, namely,

$$
\sum_{k=0}^{n}\left[p_{A}(x)\right]^{k}\left[1-p_{A}(x)\right]^{n-k}\left(\begin{array}{l}
n \\
k
\end{array}\right) \cdot\left[n-\frac{\operatorname{logit}(k / n)}{B}\right] .
$$

Note that the situation is very similar to what it was in the probit case: the first few terms and the last term of this sum are undefined!

Although the preceding discussion has concerned only the simple problem of adjusting a logistic function with a known slope to the data measured at a single intensity value, similar problems will certainly be encountered in the general case.

It is at first puzzling that the maximum-likelihood method should provide no well-defined expected value for the estimator $\hat{a}$ : After all, in the simulations, no infinities were encountered or approximations made. The puzzle is resolved when we consider that the search for the maximum of the likelihood function was restricted to the finite range $[-5,5]$ in the present simulations. Presumably, in fact, the true maximum will have been at $\pm \infty$ in a number of cases, in particular those in which all $1 \mathrm{~s}$ or all 0 s occurred at a given position. The effect of neglecting these may have been quite similar to the effect of neglecting the indefinite terms in the probit method, and biases may have arisen in a quite similar way.

(Manuscript received April 6, 1987; revision accepted for publication April 21, 1989.) 